\title{
Testing Web Services Composition: A Mapping Study
}

\author{
Hazlifah Mohd Rusli ${ }^{1}$, Suhaimi Ibrahim ${ }^{1}$ and Mazidah Puteh $^{2}$ \\ ${ }^{1}$ Universiti Teknologi Malaysia, Kuala Lumpur, Malaysia \\ ${ }^{2}$ Universiti Teknologi MARA, Shah Alam, Malaysia
}

\begin{abstract}
E-business systems are known for their frequent changes in business requirements, and traditional software development engineering approaches have difficulties in keeping up with this dynamicity. The use of service oriented architecture in software development has become popular as it provides a solution to frequent changes to business environments in a heterogeneous network. In service oriented architecture, new systems are quickly developed by combining services developed and owned by different organizations, and one way of realising this architecture is via Web services. Although much research effort has been put into the discovery, invocation and composition of services testing Web services has only begun to attract interest from both researchers and industry players. This paper aims to provide a mapping study of current Web services composition testing researches conducted by other researchers. Research papers on testing of Web services composition were gathered from various scholarly databases using provided search engines within a given period of time. The research papers were then classified according to issues addressed by them. The aim is to get a broad overview of the current state of research in Web services composition testing. By looking at the areas focused by existing researchers, gaps and untouched areas of Web services composition testing can be discovered.
\end{abstract}

Keywords: Web services, Web Services Composition, Software Testing

\section{Introduction}

Service oriented architecture is an architectural strategy that enables Web applications to be built using services, and it is estimated that $80 \%$ of software applications will be using this architecture by the year 2015 (Vaughan, 2010). Traditional software development paradigm develops software applications by gathering requirements, converting the requirements into specifications and finally implementing the specifications into executable codes. This traditional paradigm of developing software applications has difficulties in satisfying business customers needs whenever new business requirements are required of the system. In a service oriented architecture paradigm, developers are split into three entities which are the application builders (service requesters), service brokers (publishers) and service developers (providers). Service developers develop services and application builders develop new systems by combining the services. Service brokers allow developers to publish their services and application builders to find the necessary services. Therefore, instead of designing and coding new systems, system development via service oriented

Copyright (C 2011 Hazlifah Mohd Rusli, Suhaimi Ibrahim and Mazidah Puteh. This is an open access article distributed under the Creative Commons Attribution License unported 3.0, which permits unrestricted use, distribution, and reproduction in any medium, provided that original work is properly cited. Contact author: E-mail: hazlifah2@live.utm.my 
architecture is done via discovery and composition (Tsai, 2005) and one way of implementing this architecture is by using Web services.

Web services are Internet-based modular application that uses the Simple Object Access Protocol (SOAP) for communication and transfers data in XML through the Internet (Offutt and $\mathrm{Xu}, 2004$ ). It is another distributed computing technology (like CORBA, RMI, and EJB) that allows the creation of client/server application. However unlike CORBA, RMI, EJB, Web services allow easy integration and interoperability between heterogeneous applications. Web services are platform and language independent, meaning that a client program written in $\mathrm{C}$ running on Windows can communicate with a Web service application programmed in Java running on Linux. This ensures that a company's application can talk to its business partner's application, even though they are written in different languages and written on different platforms.

Recent researches study how to specify (in a formal and expressive enough language), compose (automatically), discover, secure, and ensure the correctness of Web services (Tabatabaei, 2010). Although much research attention has been given to areas of Web service discovery and composition, the same cannot be said about Web services testing. Web services testing is an important aspect of Web services as it provides a way to ensure the trustworthiness of a Web service-based system. If a client is not able to trust a service from a service provider, the client will not use it (Bai et al., 2008). Testing is also necessary to ensure that the service chosen best satisfies the user's needs and of functionalities and quality (Bai et al., 2007).

This study is interested in finding the state of research in Web services composition testing. As there are not many relevant high-quality primary studies relevant to Web services composition testing, a systematic mapping study is recommended (Kitchenham and Charters, 2007). A mapping study "involves a search of the literature to determine what sorts of studies addressing the systematic review question have been carried out, where they are published, in what databases they have been indexed, what sorts of outcomes they have assessed, and in which populations" (Petticrew and Roberts, 2006). This paper aims to provide an overview of the state of research in the area of Web services composition testing with the intention of providing future research area by looking at the gaps and untouched areas.

This paper is organized as follows. Some background information on Web services testing and composition are presented in the preliminaries in Section 2. Section 3 describes the research method in conducting the mapping study. Section 4 reports the result of the study. Section 5 describes threats to validity. Section 6 concludes the work and suggests future research direction.

\section{Preliminaries}

\section{Web Services Composition}

As mentioned earlier, application development via service oriented architecture involves searching for services that are able to fulfil the needs of the requester. However, whenever a single Web service is unable to satisfy the requirements, several Web services are composed to meet the requirements of the requester. In Web service composition, a composition schema or process definition defines the business logic of a composite Web service. A composition engine executes the business logic of a Web service composition by invoking services defined in the schema. Each composition execution is called a composition instance or process instance. Orchestration schema is a part of the composition schema that specifies the order of service invocation within a composition. BPEL and OWL-S (W3C Working Group, 2004) are two main languages created for composing Web services. BPEL is a syntax based composition language that allows manual composition of Web services whilst OWL-S is semantic based that allows a more automated approach to Web service composition. Other models used to 
compose Web services include workflows, graphs, and Petri nets.

\section{Web Services Testing}

Testing is the process of executing a program with the intent of finding errors. It involves activities such as specifying test cases, generating test data, monitoring test execution, measuring test coverage, validating test results and tracking system errors. Several research papers have been written on testing of Web services addressing areas such as testing SOAP messages, WSDL interfaces, and publish, find and bind capability. Web services composition testing involves testing the extended interaction between the service provider and requester as well as the composition schema which defines the business logic of the composite service. Existing commercial tools such as Oracle BPEL process manager and Active BPEL Designer support manual functional testing that allows testers to test BPEL descriptions and service compositions based on requirements. However existing tools/approaches are not sufficient to reveal structural errors of BPEL processes (Liu, 2008).

\section{Research Method}

\section{Research Objective}

The objective of the review paper is to summarize current state of the art Web services composition testing approaches based on the following main questions:
1. What are the research areas in Web services composition testing addressed by other studies?

2. What are number of publications per year and what is the trend?

3. Which forum type includes papers on Web services composition testing?

The above questions are instrumental in ensuring sufficient effort and time is taken to perform this mapping study.

\section{Sources of Information}

The mapping study was conducted from April to December 2010. The literature search was carried out by searching in a set of selected repositories. The repositories used were Scopus, IEEE Xplore Digital Library, ACM Digital Library, Google Scholar and Springer Link. Although the total number of papers returned was high, most of the papers were redundant. The same paper was returned by different repository search engine. Therefore, eliminating this redundancy is a necessary step in this systematic review.

\section{Search Criteria}

Based on the research questions in section 2.1 , a set of keywords and its synonyms were defined as search strings. As research in Web services composition testing is still new, no specific year range was included in the search. Table 1 describes the keywords used as well as its synonyms.

Table 1: Search Keyword and Synonyms

\begin{tabular}{|l|l|}
\hline \multicolumn{1}{|c|}{ Keyword } & \multicolumn{1}{c|}{ Synonym } \\
\hline Web service & SOA, service oriented \\
\hline Testing & Test \\
\hline Composition & Composite, BPEL \\
\hline
\end{tabular}


BPEL was used as one of the synonyms for composition as it is a prominent language for service composition. It is an XML-based specification that models Web service behavior in a business process interaction (Peltz, 2003). Using the above keywords and synonyms, a search string was created as follows:

("Web service" OR "Web services" OR "SOA") AND ("composite" OR "composition" OR "BPEL") AND ("testing" OR “test”)
The above search string was adapted for different repository search engine as the searching mechanism differs from one search engine to another.

\section{Study Selection}

A set of inclusion and exclusion criteria were defined for the selection process. These criteria were then used in the mapping study process during searching. Figure 1 illustrates the mapping study process.

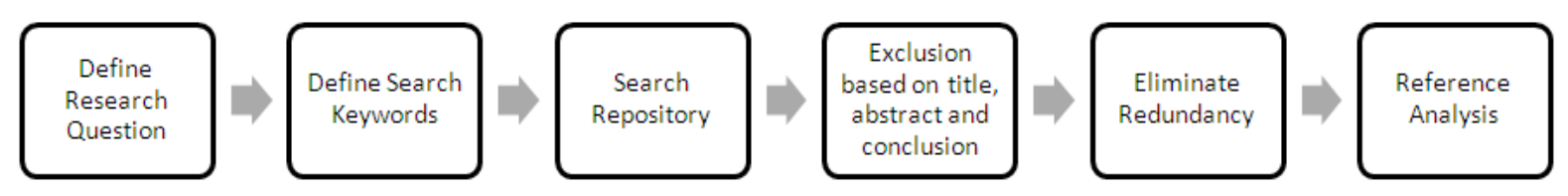

Fig 1: Mapping Study Process

The systematic review process started with defining research question as stated in section 2.1. Next step was to define the searching keyword. The keywords are listed in Table 1. Using the keywords, searching was carried out on the selected repositories using the provided search engine.

Once the list of research papers was obtained, the papers were excluded according to the title, abstract and conclusion. At the same time, the same papers returned by different repositories were removed to eliminate redundancy. Finally, reference analysis was conducted to ensure that referenced papers were not missed out. Reference analysis is important as some of the publications might have been missed out during the keywords based search using search engines and during exclusion based on title and abstract. By looking at the reference at the end of each paper, relevant papers that are missed earlier can be included in the mapping study process.

\section{Result and Discussion}

\section{WSC Testing Research Paper Areas}

A total of 45 research papers related to Web services composition testing were returned by the mapping study searching process.

The research papers were then categorized and classified into 6 groups which are survey and systematic literature reviews, test framework, test generation, regression testing and mutation testing. Figure 2 illustrates the breakdown of research papers according to the six categories. 


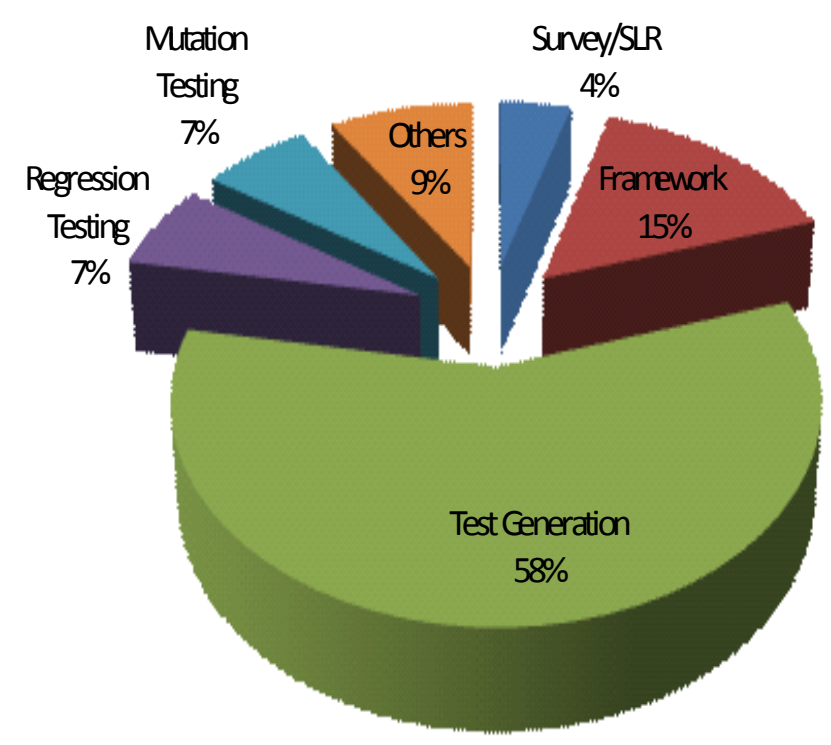

Fig 2: Breakdown of Research Paper Category

Based on Figure 2, the majority of the research papers focused on test generation issues (26 papers) followed by test framework (7 papers). Other unclassified issues contributed 4 papers. Regression testing and mutation testing studies each contributed 3 papers each. Only 2 papers were survey papers.

Figure 3 shows the number of research publications made per year for each paper category. Overall, research publications on composite Web services testing grew from just a single publication in 2004 to 14 publications in 2008. However, there is a decline in the number of papers in 2009 and as of December 2010, there are only 6 published papers. This could be due to relevant studies not yet published or indexed by digital libraries, since the literature search was conducted between April to December 2010.

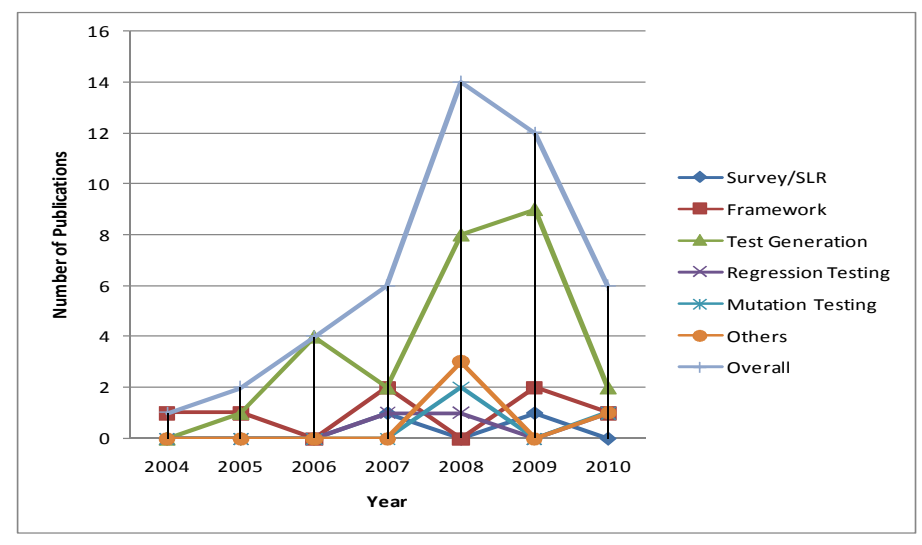

Fig 3: Number of Research Publications per Year According to Category 
Based on Figure 3, the trend is on test generation to support Web services composition testing. Research papers on test generation have the highest number of increase in publications as compared to other issues in Web services composition testing. However, issues such as regression testing and mutation testing are starting to garner interest from the research community. systematic review conducted by (Endo and Simao, 2010). The forum types are workshops, conferences and symposiums, journals and book chapter. A large percentage of the publications come from conferences and symposiums (35 papers), followed by book section (6 papers). Journals and workshops contributed 2 papers each to the overall publication category. This is illustrated in Figure 4

The publications were also classified based on different forum types, similar to

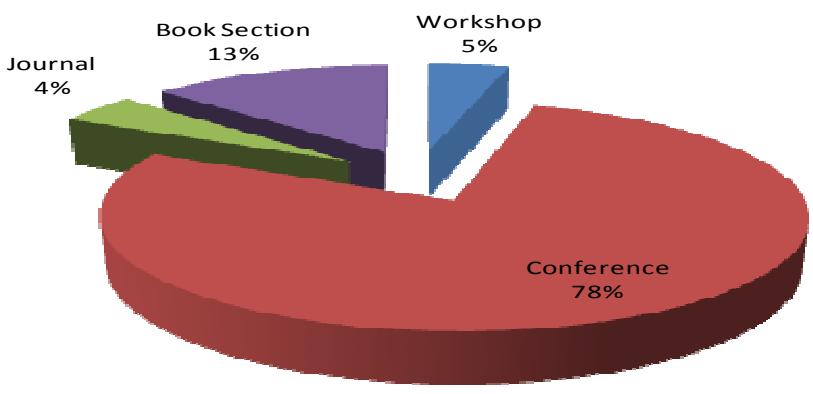

Fig 4: Breakdown of Publication's Forum Type

The high percentage of publications coming from conference and symposiums indicates that this research area is still immature and more research needs to be done to overcome issues relating to Web services composition testing. Only two publications manage to get into journals and these publications touched on test generation issues.

The following section will discuss the findings of the mapping study for each focus area as categorized in Figure 2.

\section{Survey/Systematic Review}

There are only two survey types in the literature on Web services composition testing, one by Bucchiarone et al. (2007) and the other Zakaria et al. (2009). Bucchiarone discussed web services composition testing from the aspect of orchestration and choreography and classified several papers into the two categories. For each aspect, Bucchiarone categorized them into unit and integration testing. However, his study was done in 2007 and there has been several other papers published since then. Zakaria performed a systematic review of unit testing for BPEL and provided empirical evidence on the state of BPEL studies. However, Zakaria only touched on BPEL unit testing. Both authors agree that the area of Web services testing specifically composition is still immature.

\section{Framework}

Several authors have proposed framework for Web services composition testing. Table 2 lists the proposed framework, year and framework approach. 
Table 2: Framework Development

\begin{tabular}{|c|c|c|}
\hline Author & Year & Issues Addressed \\
\hline Tsai et al. & 2004 & Group Testing \\
\hline Li et al. & 2005 & Unit Testing \\
\hline Lubke & 2007 & Unit Testing \\
\hline Karam et al. & 2007 & Abstract Workflow Based \\
\hline Kuk and Kim & 2009 & Robustness Testing \\
\hline Rauf et al. & 2009 & UML Profile Based \\
\hline Cavalli et al. & 2010 & Time Extended Finite State Machine Based \\
\hline
\end{tabular}

Tsai (2004) proposed a collaborative framework approach in testing Web service composition. The framework aims to minimize the number of testing to be carried out on composite web services for different combination of services. The approach used in the framework is based on group testing and relies on a voting mechanism. Both Lubke (2007) and Li (2005) proposed a framework for BPEL unit testing. Lubke extended Li's research by addressing issues such as test parameterization, organization and execution. Karam (2007) introduces a formal model for composed web service under test to be used in his abstract workflow-based framework. Kuk and Kim's (2009) proposed a framework that generates virtual testing environment to support robustness testing. Rauf (2009) investigated the use of UML profile in proposing a framework for BPEL Web services composition testing. Cavalli (2010) developed a testing framework that covers all phases of Web services composition development based on Time Extended Finite State Machine. In summary, the proposed frameworks supported BPEL based testing and other syntactic based Web services composition.

\section{Test Generation}

A majority of the research papers focused on test generation for Web service composition. From a total of the 42 papers, 26 papers discussed generating tests for supporting Web services composition testing. In order to analyze the test generation approaches, the following questions were defined:

1. What are the tasks involved in generating tests?

2. What are the specifications used in describing the Web service composition?

Based on the papers gathered, in general, test generation process consists of generating test model form the composition specification, generating test cases from the test model, test case execution and finally analyzing the test result. Therefore, the research papers either concentrated on test model generation or test case generation or both. Table 3 classifies the test generation approaches. 
Table 3: Classification of Test Generation Approaches

\begin{tabular}{|c|c|c|c|}
\hline Approach & Author & Year & Specification \\
\hline \multirow{13}{*}{$\begin{array}{l}\text { Test Model } \\
\text { Generation }\end{array}$} & Huang et al. & 2005 & OWL-S \\
\hline & Garcia-Fanjul & 2006 & BPEL \\
\hline & Dong et al. & 2006 & BPEL \\
\hline & Zheng et al. & 2007 & BPEL \\
\hline & Bartolini et al. & 2008 & BPEL \\
\hline & Lallali et al. & 2008 & BPEL \\
\hline & Lallali et al. & 2008 & BPEL \\
\hline & Ma et al. & 2008 & BPEL \\
\hline & Mei et al. & 2008 & BPEL \\
\hline & Dong and $\mathrm{Hu}$ & 2009 & BPEL \\
\hline & Bentakouk et al. & 2009 & BPEL \\
\hline & Mei et al & 2009 & BPEL \\
\hline & Dong & 2009 & BPEL \\
\hline \multirow[t]{3}{*}{$\begin{array}{l}\text { Test Case } \\
\text { Generation }\end{array}$} & $\begin{array}{l}\text { Lerthumpanya \& } \\
\text { Senivongse }\end{array}$ & 2008 & BPEL \\
\hline & Blanco et al. & 2009 & BPEL \\
\hline & Mao & 2009 & BPEL \\
\hline \multirow{10}{*}{$\begin{array}{l}\text { Test Model and } \\
\text { Test Case } \\
\text { Generation }\end{array}$} & Yuan et al. & 2006 & BPEL \\
\hline & Yan et al. & 2006 & BPEL \\
\hline & Wang et al. & 2007 & OWL-S \\
\hline & Lallali et al & 2008 & BPEL \\
\hline & Liu et al. & 2008 & BPEL \\
\hline & Kaschner \& Lohmann & 2009 & BPEL \\
\hline & Hou et al. & 2009 & BPEL \\
\hline & Cao et al. & 2009 & BPEL \\
\hline & Cao et al. & 2010 & BPEL \\
\hline & Cao et al. & 2010 & BPEL \\
\hline
\end{tabular}

Based on Table 3, almost all Web services composition testing involves BPEL and only two studies dealt with OWL-S. OWL-S is an upper ontology for services written using OWL and its goal is to provide general terms and properties to describe Web services. Most of the Web services composition testing studies dealt with syntactic Web services (BPEL) compared to Semantic Web services. Semantic Web services, such as OWL-S are Web services with explicit semantic annotation. All studies used test models (formal or non formal) as an input to test case generation. Extensions of Control Flow Graphs, Finite State Machines and Petri Nets were used. Test model generation research paper studied how to translate the composition specification into test models using mapping rules, and test generation research papers investigated how the derived test model can be used to generate test cases. Again, similar to studies done on framework development, the majority of the papers addressed BPEL based Web services composition. Only two papers addressed semantic Web services testing for composite Web services.

\section{Regression Testing}

Regression testing is the "selective retesting of a system or a component to verify that modifications have not caused unintended effects and that the system or component still complies with its specified requirements" (IEEE Std 610.12-1990, 1990).

Interest in regression testing is starting to emerge with two recent publications in Web services composition regression testing. This is shown in Table 4. 
Table 4: Regression Testing in Web Services Composition

\begin{tabular}{|l|c|c|c|}
\hline \multicolumn{1}{|c|}{ Author } & Year & Issues Addressed & Spec. \\
\hline Liu et al. & 2007 & Impact Analysis Rule & BPEL \\
\hline Wang et al. & 2008 & Test Case Generation & BPEL \\
\hline Yang et al. & 2010 & Fault location & BPEL \\
\hline
\end{tabular}

In his study, Liu (2007) addressed the problem of test selection in the presence of BPEL concurrent control flow by proposing a set of impact analysis rule. Wang (2008) investigated a test case generation approach in regression testing by using extended version of BPEL Flow Graph to identify changes in composite Web service and conduct change impact analysis. Yang (2010) addressed fault location issues of regression testing by inserting symbols in the test script that records testing information such as test steps number and service interface name. Again, all studies were for BPEL based composition.

\section{Mutation Testing}

Mutation testing is a technique where two or more mutant programs are executed together with the same test cases in order to determine the ability of the test cases to detect the mutants (IEEE Std 610.12-1990, 1990).Similar to regression testing research in mutation testing to support web services composition testing is also gaining attention with three recent publications. Table 5 lists down the authors involved, the approaches and specification used.

Table 5: Mutation Testing in Web Services Composition

\begin{tabular}{|c|c|c|c|}
\hline Author & Year & Issues Addressed & Specification \\
\hline Lee et al. & 2008 & $\begin{array}{c}\text { Ontology based mutant } \\
\text { operators }\end{array}$ & OWL-S \\
\hline $\begin{array}{c}\text { Wang and } \\
\text { Huang }\end{array}$ & 2008 & Mutant generator & OWL-S \\
\hline $\begin{array}{c}\text { Domínguez- } \\
\text { Jiménez et al. }\end{array}$ & 2009 & Mutant generator & BPEL \\
\hline
\end{tabular}

Lee (2008) analyzed OWL-S composite Web services fault patterns and proposed OWL-S input type mutant operators and OWL ontology mutant operators. However, the study is still in the early stage. Wang and Huang (2008) proposed a mutation testing approach based on OWL-S requirement model. Dominguez-Jimenez (2009) introduced a set of mutant operators for BPEL and proposed a framework based on genetic algorithms to generate the mutants automatically. Mutation testing is one Web services composition testing research focus area that has investigated more on the Semantic based Web services as compared to syntax based services.

\section{others}

Other research works relating to Web services composition testing include a strategy by Endo et al. (Endo et al., 2008) for Web services composition integration testing, a multi-agent test environment for BPEL based testing and test case reduction both by Dong (2008a, Dong, 2008b). Paloma-Duarte (2010) implemented a dynamic invariant generation system based on BPEL composition execution logs to generate composition invariants. All three studies were BPEL based. 


\section{Threats to Validity}

The validity of this mapping study is threatened by the following issues:

- The research papers were obtained by keyword searching and reference analysis. Exclusions were made by reading the title, abstract and conclusions. However, there is a possibility that there exist papers that were missed due to the above searching and exclusion method.

- Judgmental errors in classifying the papers into each category.

\section{Conclusion and Future Work}

This review paper has described the systematic mapping process, discussed the results of the mapping study and threats to the validity of the study. The systematic mapping process was described in terms of the research questions defined, searching keywords used, the exclusion and inclusion criteria. The results of the study was classified into several categories and analyzed. The paper has shown the areas of research within Web services composition testing that have been done by answering the questions that were defined initially. Most of the research papers are from conference and paper proceedings (76\%), which is an indication that the research area is still immature. More work need to be done in order to improve the current state of research in Web services composition testing. One research direction would be to focus on Semantic Web services composition testing. Although there are a few testing researches done on OWL-S, other Semantic Web services specification ought to be addressed as well. The author is currently investigating Web services composition testing in WSMO (W3C Working Group, 2005) which is a Semantic Web services framework.

\section{References}

Bai, X., Lee, S., Tsai, W.-T. \& Chen, Y. (2008), 'Ontology-based test modeling and partition testing of Web services,' Proceedings of the 2008 IEEE International Conference on Web Services, ISBN: 978-07695-3310-0, 23-26 September 2008, Beijing, $465-472$.
Bai, X., Wang, Y., Dai, G., Tsai, W.-T. \& Chen, Y. (2007) 'A framework for contract-based collaborative verification and validation of web services,' Component-Based Software Engineering, 4608, 258-273.

Bucchiarone, A., Melgratti, H. \& Severoni, F. (2007), 'Testing service composition,' Proceedings of the 8th Argentine Symposium on Software Engineering, 2931 August 2007, Mar del Plata, Argentina.

Cavalli, A., Cao, T. D., Mallouli, W., Martins, E., Sadovykh, A., Salva, S. \& Zaidi, F. (2010), 'WebMov: A dedicated framework for the modeling and testing of Web services composition,' Proceedings of the 2010 IEEE International Conference on Web Services, ISBN: 978-1-4244-8146-0, 5-10 July 2010, Miami, 377-384.

Domínguez-Jiménez, J., Estero-Botaro, A. \& Medina-Bulo, I. (2009), 'A framework for mutant genetic generation for WS-BPEL,' SOFSEM 2009: Theory and Practice of Computer Science, 5409, 229-240.

Dong, W. (2008a), 'Multi-agent test environment for BPEL-based Web service composition,' Proceedings of the 2008 IEEE Conference on Cybernetics and Intelligent Systems, ISBN: 978-1-4244-1673-8, 21-24 September 2008, Chengdu, 855 - 860.

Dong, W. (2008b), 'Test case reduction technique for BPEL-based testing,' Proceedings of the 2008 International Symposium on Electronic Commerce and Security, ISBN: 978-0-7695-3258-5, 3-5 August 2008, Guangzhou City, $814-817$.

Endo, A. T., Da Silva Simao, A., De Souza, S. \& De Souza, P. (2008), 'Web services composition testing: a strategy based on structural testing of parallel programs,' Proceedings of the Testing: Academic \& Industrial Conference Practice and Research Techniques, ISBN: 978-0-76953383-4, 29-31 August 2008, Windsor, 3 12.

Endo, A. T. \& Simao, A. D. S. (2010), “Formal testing approaches for service-oriented architectures and Web services: A systematic review," [Online], [Retrieved August 22, 2010], 
http://citeseerx.ist.psu.edu/viewdoc/sum mary?doi=10.1.1.169.9170

IEEE STD 610.12-1990 (1990), “IEEE Standard Glossary of Software Engineering Terminology"

Karam, M., Safa, H. \& Artail, H. (2007), 'An abstract workflow-based framework for testing composed Web services,' Proceedings of the 2007 IEEE/ACS International Conference on Computer Systems and Applications, ISBN: 1-42441030-4, 13-16 May 2007, Amman, 901 908.

Kitchenham, B. \& Charters, S. (2007), "Guidelines for performing systematic literature reviews in software engineering," [Online], [Retrieved August 21, 2010], http://www.citeulike.org/user/sebastianp y/article/3955888

Kuk, S. H. \& Kim, H. S. (2009), 'Robustness testing framework for Web services composition,' Proceedings of the 2009 Services Computing Conference, ISBN: 9781-4244-5338-2, 7-11 Dec. 2009, Singapore, $319-324$

Lee, S., Bai, X. \& Chen, Y. (2008), 'Automatic mutation testing and simulation on OWL-S specified Web services,' Proceedings of the 41st Annual Simulation Symposium, ISBN: 0-7695-3143-1, 13-16 April 2008, Ottawa, 149 - 156.

Li, Z., Sun, W., Jiang, Z. B. \& Zhang, X. (2005), 'BPEL4WS Unit testing: Framework and implementation,' Proceedings of the 2005 IEEE International Conference on Web Services, ISBN: 0-7695-2409-5, 11-15 July 2005, $103-110$.

Liu, H., Li, Z., Zhu, J. \& Tan, H. (2007), 'Business process regression testing,' Service-Oriented Computing, 4749, 157168.

Liu, C. H., Chen, S. L. \& Li, X. Y. (2008), 'A WS-BPEL based structural testing approach for Web service compositions,' Proceedings of the 2008 IEEE International Symposium on Service-Oriented System Engineering, ISBN: 978-0-7695-3499-2, 1819 December 2008, Jhongli, 135-141.
Lübke, D. (2007), 'Unit testing BPEL compositions,' Test and Analysis of Web Services, $149-171$

Offutt, J. \& Xu, W. (2004), 'Generating test cases for Web services using data perturbation,' ACM SIGSOFT Software Engineering Notes, 29, 1-10.

Palomo-Duarte, M., Garcıa-Domınguez, A., Medina-Bulo, I., Alvarez-Ayllon, A. and Santacruz, J. (2010), 'Takuan: A tool for WS-BPEL composition testing using dynamic invariant generation,' Proceedings of 10th International Conference on Web Engineering, 5-9 July 2010, Vienna, 531534.

Peltz, C. (2003), 'Web services orchestration and choreography,' Computer, 36, 46-52.

Petticrew, M. \& Roberts, H. (2006) Systematic Reviews in the Social Sciences: A Practical Guide, Blackwell Publishing, Malden, Massachusetts.

Rauf, I., Iqbal, M. Z. Z. \& Malik, Z. I. (2009), 'Model based testing of Web service composition using UML profile,' Proceedings of the 2nd Workshop on Model-based Testing in Practice, ISSN 0929-0672, 23-26 June 2009, The Netherlands, 75 - 84 .

Tabatabaei, S. G. H, Dastjerdi, A. V., Wan Kadir, W. M. N., Ibrahim, S. \& Sarafian, E. (2010) 'Security conscious AI-planningbased composition of semantic Web services,' International Journal of Web Information Systems, 6(3), 203-229.

Tsai, W.T. (2005), 'Service-Oriented System Engineering: A new paradigm,' Proceedings of 2005 IEEE International Workshop on Service-Oriented System Engineering, ISBN: 0-7695-2438-9, 20-21 October 2005, Beijing, 3-6.

Tsai, W. T., Chen, Y., Paul, R., Liao, N. \& Huang, H. (2004), 'Cooperative and group testing in verification of dynamic composite Web services,' Proceedings of the 28th Annual International Computer Software and Applications Conference, ISBN: 0-7695-2209-2, 28-30 September 2004, $170-173$. 
Vaughan, J., (2010), “Gartner: SOA will be like electricity for architects looking toward cloud computing," SOA News. [Online], [Retrieved November 23, 2010], http://searchsoa.techtarget.com/news/art icle/0,289142,sid26_gci1523670,00.html

W3C Working Group (2004), “OWL-S: Semantic Markup for Web Services" [Online], [Retrieved October 20, 2010], http://www.w3.org/Submission/OWL-S/

W3C Working Group (2005), "Web Service Modeling Ontology (WSMO)" [Online], [Retrieved October 20, 2010], http://www.w3.org/Submission/WSMO/

Wang, D., Li, B. \& Cai, J. (2008), 'Regression testing of composite service: An XBFGbased approach,' Proceedings of the IEEE Congress on Services Part II, ISBN: 978-07695-3313-1, 23-26 September 2008, Beijing, $112-119$.

Wang, R. \& Huang, N. (2008), 'Requirement model-based mutation testing for Web service,' Proceedings of the 4th International Conference on Next Generation Web Services Practices, ISBN: 978-0-7695-3455-8, 20-22 October 2008, Seoul, $71-76$.

Yang, B., Wu, J., Liu, C. and Xu, L. (2010), 'A regression testing method for composite Web service,' Proceedings of the 2010 International Conference on Biomedical Engineering and Computer Science, ISBN: 978-1-4244-5315-3, 23-25 April 2010, Wuhan, 1-5.

Zakaria, Z., Atan, R., Ghani, A. A. A. \& Sani, N. F. M. (2009), 'Unit testing approaches for BPEL: A systematic review,' Proceedings of the 2009 Asia-Pacific Software Engineering Conference, ISBN: 978-0-7695-3909-6, 1-3 Dec. 2009, Penang, 316 - 322. 